\title{
Associations between DNA methylation and telomere length during early life: insight from wild zebra finches (Taeniopygia guttata)
}

\author{
Elizabeth Sheldon ${ }^{1}$, Riccardo Ton ${ }^{2}$, Winnie Boner ${ }^{3}$, Pat Monaghan ${ }^{3}$, Shirley Raveh ${ }^{3}$, \\ Aaron Schrey ${ }^{4}$, and Simon Griffith ${ }^{2}$ \\ ${ }^{1}$ University of South Florida \\ ${ }^{2}$ Macquarie University \\ ${ }^{3}$ Glasgow University \\ ${ }^{4}$ Georgia Southern University
}

March 31, 2021

\begin{abstract}
Telomere length and DNA methylation (DNAm) are two promising biomarkers of biological age. Environmental factors and life history traits are known to affect variation in both these biomarkers, especially during early life, yet surprisingly little is known about their reciprocal association. Here, we present the first study on a natural population to explore how variation in DNAm, growth rate and early-life conditions are associated with telomere length changes during development. We tested these associations by collecting data from wild, nestling zebra finches in the Australian desert. We found that increases in the level of DNAm were negatively correlated with telomere length changes across early life. We also confirm previously documented effects of post hatch growth rate and clutch size on telomere length in a natural ecological context for a species that has been extensively studied in the laboratory. However, we did not detect any effect of ambient temperature during developmental on telomere dynamics. We also found that the absolute telomere length of wild zebra finches, measured using the in-gel TRF method, was similar to that of captive birds. Our findings highlight exciting new opportunities to link and disentangle potential relationships between environmental, epigenetic and telomere length dynamics during early life.
\end{abstract}

\section{INTRODUCTION}

Telomeres are highly conserved, non-coding DNA sequences that form protective caps at the end of eukaryotic chromosomes (Blackburn,1991, Blackburn \& Epel 2012). In the absence of telomerase (a reverse transcriptase that adds telomeric repeats de novo after each cell division), telomeres shorten with each round of cell division (Harley, Futcher, \& Greider, 1990). When a critical length is lost, telomeres become dysfunctional and cells enter a state of replicative senescence (Hornsby, 2003; Verdun \& Karlseder, 2007, see also Victorelli \& Passos, (2017) for length independent damage to telomeres triggering cell senescence). The accumulation of senescent cells is known to contribute to age-related declines in tissue and organ function (Wong et al., 2003). Accordingly, within species, relatively short telomeres and accelerated rates of telomere shortening have been associated with fitness costs, predominantly via reduced lifespan, at the individual level (Monaghan, 2010; Eastwood et al., 2019; Boonekamp, Mulder, Salomons, Dijkstra, \& Verhulst, 2014, Wilbourn et al., 2018). Given the relationship with lifespan, telomeres have been widely used as biomarkers of biological age (Jylhava, Pedersen, \& Hagg, 2017). This has also led to a recent focus in understanding how inter-individual variation in telomere length arises. Early life telomere length is partly determined by genetic factors (Olsson et al., 2011; Dugdale \& Richardson 2018), however, accumulating evidence suggests that environmental cues also impact telomere length dynamics across the life course (Kotrschal, Ilmonen, Penn, 2007; Vedder, Verhulst, Zuidersma, \& Bouwhuis, 2018; Dupoue et al., 2017; Boonekamp, et al., 2014). Information on the relationship between environmental and telomere length variation is steadily increasing, yet surprisingly 
little is known about whether other DNA modifications mirror telomere dynamics. Insight on how telomere length can vary in concert with other genetic traits can expand our understanding of DNA based biological markers of aging, and of physiological reactions to environmental change.

DNA methylation (DNAm) is an epigenetic mechanism that appears to be an important component of telomere length regulation (Blasco, 2007), and is also considered a promising biological clock (Horvath \& Raj 2018). Yet, the relationship between DNAm and telomere length has not yet been explored in an ecological context in any species. DNAm usually refers to the addition of a methyl group to a cytosine base at a CG dinucleotide (a 'CpG' site) on the DNA sequence, but can occur at other sites in different taxa (Anglers et al., 2010). When DNAm occurs at a CpG site close to a gene regulatory region, it can modulate phenotypic variation through its effects on gene expression. Evidence suggests that DNAm could be involved in two, key telomere regulatory processes; those involving telomerase (Buxton et al., 2014), and 'alternative mechanisms' relying on homologous recombination between telomeric sequences such as alternative lengthening of telomeres (Gonzalo et al., 2006). Indeed, while telomeres themselves do not contain $\mathrm{CpG}$ sites, decreases in global and subtelomeric DNAm are known to be concomitant with increased homologous recombination between telomeric sequences and dramatically elongated telomeres in mouse cells (Gonzalo et al., 2006). Additionally, a negative relationship between telomere length and genome-wide (Lee et al., 2019) and gene-specific (Lee et al., 2019; Buxton et al., 2014) DNAm has been described in humans (however, this relationship may be complicated as a positive relationship has been detected in a different correlative study (Dong et al., 2018)). Evidence also suggests that short telomeres have specific epigenetic marks that may facilitate their preferential elongation (Hemann, Strong, Hao, \& Greider, 2001; de Lange, 2005). These studies suggest the existence of a direct relationship between DNAm and telomere length. However, there is also plenty of scope for indirect relationships between DNAm and telomere length to occur given that they are associated with a range of the same biological and ecological factors (Figure 1) and are also both independently identified as molecular measures of age and ageing (Lu et al., 2019; Banszerus, Vetter, Salewsky, König \& Demuth, 2019).

To date, surprisingly little work has explored the potential links between telomere length and DNAm, although a few studies have indicated some conceptual connections (Horvath et al 2018; Blasco, 2007; Figure 1). DNAm and telomere length are highly responsive to environmental cues (Feil \& Fraga, 2012; Monaghan, 2014), particularly during early life (Watson, Bolton \& Monaghan, 2019; Boonekamp et al., 2014). For example, studies have detected associations between DNAm or telomere length and clutch/brood size (Noguera \& Velando, 2020; Jimeno, Hau, Gomez-Diaz, \& Verhulst, 2019; Sheldon, Schrey, Ragsdale \& Griffith 2018; Nettle et al., 2016; Reichert et al., 2014; Costanzo et al., 2016; Boonekamp et al., 2014); ambient temperature (Stier, Metcalfe \& Monaghan, 2020; Sheldon, Schrey, Hurley, \& Griffith, 2020; Yan et al., 2015); and body size/growth rate (Young et al., 2017; Vedder et al., 2018). It is therefore important to account for these influences when testing the association between DNAm and telomere length. The present study is the first examination, to date, of the relationship between DNAm and telomere length dynamics in a species in the wild in which individuals are exposed to natural variation in early life conditions. Specifically, we collected longitudinal measures of telomere length (using qPCR) and genome-wide levels of DNAm (using methylation sensitive- amplification fragment length polymorphisms (MS-AFLP)) across the early life of wild zebra finches (Taeniopygia guttata). Although MS-AFLP analyses do not provide inference on the functional, gene regulatory consequences of DNAm differences, they do provide a useful tool to compare the relationship between telomere length dynamics and changes in the percentage of a consistent subset of CpG loci that are methylated among individuals (Schrey et al., 2013). Our study aims to provide an initial exploration into the relationship between early life DNAm changes and telomere length changes in wild animals as well as potential associations with ambient temperature and life history effects. This can establish a base for future research to link and/or disentangle the relationship between early life conditions, DNA based biomarkers of age, and individual fitness parameters.

In addition to considering the links between telomere length and DNAm, our study is also of value in providing the first investigation of telomere length dynamics in wild zebra finches Taenopygia guttata . Characterisations of telomere dynamics in the wild have become increasingly common for a wide range of bird 
species (Supplementary Table 1a and 1b). In contrast, laboratory studies on birds are largely performed using the zebra finch, in which paradoxically, telomere dynamics have yet to be explored in the wild (Supplementary Table 1a). Studies of ecologically relevant populations of wild zebra finches are thus of value in helping to contextualize and interpret the controlled laboratory tests that have made such a significant contribution to our understanding of telomere biology in birds.

\section{MATERIALS AND METHODS}

\section{Field work and blood sampling}

Fieldwork was conducted at Fowlers Gap Arid Research Station, in far-western New South Wales $\left(31^{\circ} 05^{\prime} \mathrm{S}\right.$, ' $141^{\circ} 42^{\prime} \mathrm{E}$ ) during the main part of the Austral breeding season (August-December) in 2016. Data were collected from zebra finch nestlings in nest boxes in their natural habitat at 'Fowlers Gap' (details regarding field site characteristics can be found in Griffith, Pryke, \& Mariette, 2008). Given the mobility of the species in the wild, we were working with a population in which most adults were not banded, and we were unable to ascertain parents' age or reproductive history. Nest boxes were monitored periodically (every 2 days) during the nest-building stage, and after the first egg was laid nest boxes were monitored each morning until the entire clutch was laid, enabling us to ascertain clutch size and projected hatch date. In the wild, we have previously shown that parent zebra finches only initiate incubation on the day that the last egg is laid (Gilby, Mainwaring, \& Griffith, 2013). Consequently, we used the day of the last laid egg to represent the first day of embryonic development and the usual incubation period of $\sim 12$ days after the onset of incubation, to predict clutch hatch date. Two days prior to a clutch's estimated hatch date, we monitored the nest three times per day between 06.00 and 17.00; our first nest check was at $\sim 06.00$, our second nest check was at $\sim 11.30$ and our third nest check was at $\sim 17.00$, which allowed us to identify the hatch date of each nestling. The hatch date was used to calculate the post-hatch age of each nestling (in days). Synchronous hatching meant that we could not tie nestlings to specific eggs, we were unable to account for the order in which eggs/nestlings were produced, something that has been shown to impact telomere dynamics in the zebra finch (Noguera, Metcalfe, Reichert \& Monaghan, 2016).

To measure ambient developmental temperature, we obtained hourly atmospheric temperature data in the shade (i.e. air temperature) from the Australian Bureau of Meteorology's automated weather station at Fowlers Gap, located within $20 \mathrm{~km}$ of the study site. We calculated the mean temperature during each day of zebra finch development using hourly data from $7 \mathrm{am}$ to $7 \mathrm{pm}$. These data represent the period of the day when the adults are periodically away from the nest foraging together (Mariette \& Griffith 2015), and the nestlings (or embryos) are therefore subject to the greatest exposure to ambient (rather than brooding/ incubation) temperatures; the duration of nestling exposure to ambient temperature increases as the nestlings grow, and parents reduce brooding. The daily temperature values were then averaged from day 3 to day 11 of post-hatch development (prior to day 3 , the ectothermic nestlings are typically brooded or incubated for long periods during the day, thus are not exposed to ambient temperatures, day 3 was also the age at which the earliest blood sample was taken, see below). During our sampling period (Aug-Nov), average temperatures across post-hatch development ranged from $14.6-27.6^{\circ} \mathrm{C}$ (average $20.3^{\circ} \mathrm{C}$, SEM: 0.295). At day 3 a small patch of down feathers was trimmed from a different area on each nestling allowing us to identify individual nestlings throughout development. Trimming the small patch of hair (approximately $0.5 \mathrm{~cm}^{2}$ ) from each nestling was unlikely to impose thermoregulatory costs.

At day 3 and day 11, we extracted blood $(<20 \mu l)$ from the metatarsal vein of younger (day 3$)$ nestlings, and the brachial vein of older (day 11) nestlings with a hypodermic needle and capillary tube. Blood was preserved in $0.5 \mathrm{ml}$ of $95 \%$ ethanol and stored at room temperature in an Eppendorf tube that was labelled with a unique ID. Growth rate (a measure of cell proliferation) can affect telomere dynamics (Boonekamp et al., 2020; Ringsby et al., 2015). To account for variation in growth rate between individual nestlings and broods we measured tarsus length (a reliable metric of body size) at day 3 and day 11 using digital calipers. The associated effects of clutch and brood size (i.e. parental care and resource acquisition) can also affect telomere length (Costanzo et al., 2016; Boonekamp et al., 2014) and DNAm changes (Sheldon et al., 2018), thus, we included clutch and brood size in our model. We collected data on the clutch size of each 
nest daily from the day of first lay to the onset of incubation, and on the two days prior to the estimated day of hatch (clutch size ranged from 2 to 11 eggs). We collected data on the brood size of each nest on the day the entire clutch hatched, day 3, and day 11 of post-hatch development (brood size ranged from 2 to 8 nestlings). Due to egg/nestling mortality, clutch/brood size often varied across development, thus we averaged the clutch/brood size of each nest across the two time-points.

\section{Sample size}

Two blood samples were available for analysis from 91 zebra finches from 62 nests - a total of 182 samples. $73 \%$ of these samples yielded enough DNA for assaying telomere length successfully using qPCR - a total of 133 samples from 83 individuals from 57 nests, 50 of these individuals from 38 nests were measured successfully for telomere length at both day 3 and day 11 . Of the 83 individuals analysed successfully for telomere length, 78 individuals from 60 nests, a total of 106 samples, were analysed successfully for MSAFLP. 28 of these individuals from 23 nests were measured successfully for MS-AFLP at both day 3 andday 11. Sample failures during qPCR analysis were generally due to an insufficient DNA yield from a small initial blood sample. Sample failures during MS-AFLP analysis were generally due to the sensitivity of the MS-AFLP protocol to DNA concentration during the restriction digest step. Due to logistical constraints during field work, tarsus length and/or clutch size was not recorded for six nestlings from five nests, leading to the final sample sizes reported in Table 1 and 2.

\section{DNA methylation measurements}

The MS-AFLP protocol used modifies the standard AFLP protocol by substituting the MseI enzyme with the methylation-sensitive isoschizomeric enzymes MspI and HpaII (New England Biolabs). See Salmon et al., (2008) for further details on the MS-AFLP method. Together, four types of variation can be scored: Type 1 is when both enzymes cut at the restriction site and indicates no methylation, Type 2 is when MspI does cut and HpaII does not cut, indicating the restriction site has a methylated internal cytosine C; Type 3 is when MspI does not cut and HpaII does cut indicating the restriction site has hemi-methylation; and Type 4 is when neither enzyme cuts indicating either both cytosines are methylated or the restriction site has mutated (Richards, Schrey \& Pigliucci, 2012) (conservatively, we treated Type 4 as missing data because the underlying methylation state cannot be determined; for example see Richards et al., 2012).

We performed MS-AFLP following the protocol used by Richards et al., (2012). For the MS-AFLP analysis, DNA was extracted using the Gentra Puregene tissue kit (Qiagen, Valencia, CA, USA) and was stored in 40 $\mu \mathrm{l}$ of TE buffer. For both day 3 and day 11 samples, we digested approximately 250ng of genomic DNA at $37^{\circ} \mathrm{C}$ for $3 \mathrm{~h}$ in paired reactions: one with EcoRI and MspI, the other with EcoRI and HpaII. We immediately followed the restriction digest with adaptor ligation with EcoRI and MspI/HpaII adaptors at 16-20 h at $16^{0}$ C (Supplementary Material Table 2, all primer and adapter sequences). After adaptor ligation, we conducted pre-selective PCR with EcoRI+1, MspI/HpaII+0 pre-selective primers (Supplementary Material Table 2) at the following PCR conditions: $75^{\mathrm{o}} \mathrm{C}$ for $2 \mathrm{~min} ; 20$ cycles of $94^{\mathrm{O}} \mathrm{C}$ for $30 \mathrm{~s}, 56^{\mathrm{O}} \mathrm{C}$ for $30 \mathrm{~s}, 75^{\mathrm{O}} \mathrm{C}$ for 2 min, final extension at $60^{\circ} \mathrm{C}$ for $30 \mathrm{~min}$ and $4^{\circ} \mathrm{C}$ hold. Following pre-selective PCR, we conducted selective PCR by multiplexing 6-FAM fluorescently labelled EcoRI+AGC primers with HEX fluorescently labelled EcoRI+ACG primers and unlabeled primers HpaII/MspI+TCAT (Supplemental Table 2) at the following PCR conditions $94^{\circ} \mathrm{C}$ for $2 \mathrm{~min}, 8$ cycles of $94^{\circ} \mathrm{C} 30 \mathrm{~s}, 65^{\circ} \mathrm{C} 30 \mathrm{~s} 72^{\circ} \mathrm{C} 2 \mathrm{~min}$ (dropping the annealing temperature $1^{\mathrm{O}}$ each cycle), 31 cycles of $94^{\mathrm{O}} \mathrm{C} 30 \mathrm{~s}, 56^{\mathrm{O}} \mathrm{C} 30 \mathrm{~s} 72^{\mathrm{O}} \mathrm{C} 2 \mathrm{~min}$, final extension of $60^{\circ} \mathrm{C} 5 \mathrm{~min}$ and a $4^{\mathrm{O}} \mathrm{C}$ hold. We sent the selective PCR products to Macrogen Facilities (South Korea) for fragment analysis on an ABI 3130XL.

We used PEAKSCANNER v 1.0 (Applied Biosystems) to analyse resultant gel files and define fragment sizes, and RAWGENO (Arrigo et al., 2012) to define bands. We pooled data into two categories: methylated (Type 2 and Type 3 ) or not methylated (Type 1). Throughout, we refer to a MS-AFLP locus to indicate a particular sized band resolved in the selective PCR.

To ensure scores were consistent, we validated our MS-AFLP results by duplicating the entire protocol for 30 random individuals. We identified bands that consistently occurred, and we eliminated bands that were 
inconsistently amplified or occurred at highly variable intensities. We conducted all analyses using a binary haplotype-binding pattern (methylated 1, not methylated 0) for 92 verified consistent CpG sites between 50 and 500 base pairs for each nestling. We calculated percentage of genome wide DNAm as the proportion of the 92 loci that were methylated for each sample, we refer to this throughout as DNAm.

\section{Telomere measurements}

Genomic DNA (gDNA) was extracted using the NucleoSpin blood kit (Macherey-Nagel) with some minor modifications. $2 \mu \mathrm{l}$ of whole blood was removed from the sample tube and allowed to air-dry, to evaporate the ethanol, and subsequently added to $198 \mu \mathrm{l}$ of PBS. From this stage, the manufacturer's protocol was followed, with the gDNA eluted into $35 \mu \mathrm{l}$ of BE buffer. The quantity and purity of the gDNA was measured on a Nanodrop 8000 and all samples were within the accepted parameters; $\mathrm{A}_{260 / 280}$ [?]1.7, $\mathrm{A}_{260 / 230}$ [?] 1.8 (Thermo Fisher). The gDNA was stored at $-20^{\circ} \mathrm{C}$ until telomere length analysis was performed.

Telomere length was assayed using the qPCR method as described previously (Criscuolo 2009). Briefly, the telomere length of each sample was measured by determining the ratio $(\mathrm{T}: \mathrm{S})$ of telomere repeat copy number (T) to a single copy or non-variant control gene (S), relative to a five pooled DNA reference sample from wild zebra finches at day 11 that was run on all plates. For this study, Glyceraldehyde-3-phosphate dehydrogenase (GAPDH) was used as the control gene. A standard curve (6 serial dilutions of zebra finch gDNA from 40 to $1.25 \mathrm{ng} /$ well) was also included on each plate and all samples (ran in triplicate) fell within standard curve boundaries. Mean reaction efficiencies were within the acceptable range for telomere and control gene (mean \pm SE, TEL: $97.43 \pm 4.54 \%$; GAPDH: $87.73 \pm 3.75 \%$ ). The average inter-plate variation of the $\mathrm{Ct}$ values was 1.47 for the telomere assay and 2.6 for the GAPDH assay. Intra-plate coefficient of variation for the telomere and GAPDH assays for the raw $\mathrm{Ct}$ values were 12.44 and 27.08 respectively. The average intra-plate variation of the $\mathrm{Ct}$ values was 0.74 for the telomere assay and 0.47 for the GAPDH assay.

Telomere length measurements were calculated using the method by Pfaffl (2001). The mean values were used to calculate the telomere length (T:S ratio) using the formula: $\left((1+\mathrm{E} \text { telomere })^{\wedge} \Delta \mathrm{Cq}\right.$ telomere (control - sample) / (1 + E GAPDH $)^{\wedge} \Delta \mathrm{Cq}$ GAPDH (control - sample) $)$.

In addition we had sufficient DNA from a small number of 11 day samples (5) that enabled us to measure absolute telomere length using the in-gel TRF method (see Nussey et al., 2014 for details of this method). This enabled us to compare telomere length in wild and captive zebra finches.

\section{Statistical analysis}

All statistical analyses were performed in R 4.0.3 (R Core Team, 2017). Linear mixed models (LMMs) were run using the package lmer (Bates, Machler, Bolker, \& Walker , 2015), with package lmerTest (Kuznetsova, Brockhoff, \& Christensen 2016) to calculate degrees of freedom and $p$-values. We calculated marginal $\mathrm{R}^{2}$ values for the LMMs using the method described by Nakagawa and Schielzeth (2013). We initially performed a series of linear regression analyses to test for possible effects of body size on telomere length, possible effects of body size on percent DNAm changes and possible effects of temperature on growth rate/body size (Supplementary Analysis 1). We also performed a LMM to examine the effect of baseline telomere length (at day 3) on change in telomere length after adjusting for the effects of regression to the mean (RTM) (Verhulst, Aviv, Benetos, Berenson, \& Kark, 2013; Supplementary Analysis 2).

In a first, longitudinal analysis, we used a LMM (Model 1) to explore the effects of temperature, clutch size, change in DNAm and change in tarsus (growth from day 3 to day 11) (all as fixed effects) on the rate of changes in telomere length from day 3 to day 11 (dependent variable). Our preliminary analysis (Supplementary analysis 2c) detected a negative correlation between tarsus length at day 3 and telomere length at day 3. Therefore, we also included tarsus length at day 3 as a fixed effect to account for potential effects of body size on telomere length changes. Nest ID was included as a random effect in this model to control for possible familial/genetic effects, and the repeated sampling of the same individuals within a brood. Only individuals with data for both day 3 and day 11 were included in this longitudinal analysis. Change between day 3 and day 11 was calculated using the equation $($ change $=($ value at day $11-$ value at 
day 3)). Models were built on the biological variables of interest.

In our cross-sectional analyses, we performed two LMMs to examine a) Model 2: the effects of temperature, clutch size, DNAm at day 3, and tarsus length at day 3 (fixed effects) on telomere length at day 3 (the dependent variable), and b) Model 3: the effects of temperature, brood size, DNAm at day 11, and tarsus length at day 11 (fixed effects) on telomere length at day 11. We accounted for genetic and/or familial effects by including Nest ID as a random effect.

\section{Literature search}

We compiled a list of peer-reviewed scientific publications on avian telomere dynamics from 2000 to 2020 using Google Scholar and Web of Science as online search engines. We simultaneously used "telomere", "zebra finch", and "birds" as keywords, including " " in front of every word to consider also potential synonyms. A further search was performed also using the same keywords individually to cross check the comprehensiveness of our results. We performed the web search first considering the entire 20-year interval and then on a year-by-year basis. We only recorded studies published in peer reviewed journals and excluded thesis', reviews, metanalyses and preprints. We divided our total sample into two categories: 1) telomere studies using the zebra finch, and 2) telomere studies using any species other than zebra finch. For each category we listed the studies in ascending chronological order, and for multiple publications within each year we listed authors in alphabetical order. The results of our literature search are summarized in Table 1 of Supplementary Materials.

\section{RESULTS}

The telomere length of the five wild zebra finches measured using the in-gel TRF method was similar to that of captive bred birds (range 12.2-13.59kb, mean $12.77 \mathrm{~kb}+/-0.24$. Overall, there was no change in telomere length between day 3 and day 11 of post-hatch development, however on average Mean telomere length was 0.84 (range: 0.29-2.59, SEM: 0.059) on day 3, and 0.74 (range: 0.44-2.16, SEM: 0.041) on day 11. This decrease in telomere length was not statistically significant (paired t-test: $t_{49}=1.59, p=0.116$ ) (Figure 2 ). Thirty-three individuals showed a reduction in telomere length between day 3 and day 11 post-hatch, while 17 were found to have telomeres that increased in length. Telomere length decreased by an average of 0.28 (range: -0.64 to -0.02 , SEM: 0.03 ), and increased by 0.25 (range: $<0.01$ to 1.92 , SEM: 0.16 ).

Our MS-AFLP analyses verified 92 consistent CpG sites between 50 and 500 base pairs. (\%) DNAm was $29.2 \%$ at day 3 (Standard deviation $(\mathrm{SD})=6.5)$, and $34.9 \%$ at day $11(\mathrm{SD}=8.3)$. We found a significant, negative relationship between changes in the level (\%) of DNAm across these $92 \mathrm{CpG}$ sites and change in telomere length between day 3 and day 11 (Table 1, Figure 3a). We also found a significant, negative relationship between change in tarsus length and change in telomere length between day 3 and day 11 (Table 1, Figure $3 \mathrm{~b}$ ). Tarsus length at day 3 , brood size and post-hatch ambient temperature did not significantly affect change in telomere length (Table 1). The fixed factors explained $43.73 \%$ (Marginal $\mathrm{R}^{2}=0.437$ ), while the random effect of natal nest ID explained $<1 \%$ of the variance in the data (Conditional $\mathrm{R}^{2}=0.441$; this value describes the proportion of variance explained by both the fixed and the random factor, thus most variance in this model was explained by the fixed factors) (Table 1)).

In our second LMM we detected a significant, positive association between clutch size and telomere length at day 3 (i.e., larger clutches were associated with longer telomeres at day 3 (Table 2)), while percent DNAm at day 3 and other fixed factors (pre-hatch ambient temperature and tarsus length at day 3 ) were not related to telomere length. In this model, the fixed factors explained $28.57 \%$ of the variance in telomere length at day 3 (Marginal $\mathrm{R}^{2}=0.285$ ), while the random effect of natal nest ID explained $40.19 \%$ of variance in telomere length at day 3 (Conditional $\mathrm{R}^{2}=0.687$ ) (Table 2 ).

In our third LMM, DNAm at day 11 was not related to telomere length at day 11, and, similarly none of the other fixed factors (brood size, temperature and tarsus length at day 11) included in our model appeared to influence telomere length at day 11 (Table 3). The fixed factors in our model (Table 3), explained $8.63 \%$ of the variance in the data (Marginal $\mathrm{R}^{2}=0.086$ ), while the random effect of natal nest ID explained $59.43 \%$ of 
variance in telomere length at day 11 post-hatch (Conditional $\mathrm{R}^{2}=0.6807$ ) (Table 3 ). After visual inspection of figures $2,3 \mathrm{a}$ and $3 \mathrm{~b}$, we identified an individual with a particularly high telomere length value. We re-ran all our models after excluding this anomalous data point, however, excluding this data point did not alter our results significantly. Since there was no biological reason to omit this individual from the data set it was included in our final analyses.

\section{DISCUSSION}

Our study is the first investigation of the relationship between telomere length and DNAm dynamics in wild animals exposed to naturally variable early life conditions. In our longitudinal analysis we found a negative relationship between individual changes in telomere length and changes in the level of methylated CpG loci (92 CpG loci in our study) across early life, such that individuals with accelerated rates of telomere shortening were associated with increases in DNAm levels. The observational nature of our field data means that we were unable to establish whether the negative relationship between telomere length and DNAm changes reflected coincidental or causal effects. That is, whether DNAm and telomere length respond comparably to the same intrinsic/extrinsic conditions experienced across early life and/or whether potential regulatory effects between DNAm and telomere length may exist. How changes in telomere length and DNAm relate to each other and respond to early life conditions warrants further investigation since it may help identify potentially distinct aspects of biological age that DNAm and telomere length reflect. It is important to note that while variation in DNAm levels may reflect variation in the early life environment (Angers, Castonguay, \& Massicotte, 2010; Sheldon et al., 2018; 2020; Makinen, van Oers, Eeva, Laine \& Ruuskanen, 2020), the relevance of early-life, 'genome-wide' hypo or hyper methylation in the context of biological age remains unclear. Thus, while our MS-AFLP analyses are useful to compare DNAm and telomere length associations in different environments, next-generation sequencing data (used to generate an 'epigenetic clock') are necessary to compare DNAm and telomere length predictors of biological age in different environments (Horvath \& Raj 2018; Banszerus et al., 2019). We did not detect a relationship between cross-sectional measures of DNAm and telomere length at either day 3 or day 11 post-hatch. However, telomere length at day 3 was weakly, but significantly positively associated with clutch size. Given this relationship was positive rather than negative, it suggests that the within clutch decline in telomere length across the laying order that has been seen in other studies did not have much effect here. The positive relationship could perhaps be due to parental age if older parents had a reduced clutch size and their offspring inherited shorter telomere lengths or lost more up to this point (Monaghan and Metcalfe, 2019).

Evidence is accumulating to suggest that longitudinal measures of within-individual change in telomere length may represent a more useful biomarker of ageing/individual condition than static, cross-sectional telomere length measures (Wood and Young, 2019; Boonekamp et al., 2014; Tricola et al., 2018; Wilbourn et al., 2018. However, to our knowledge, no other study has conducted longitudinal analyses on the relationship between within-individual change in telomere length and DNAm across early development, in any species. Although it may be expected that factors associated with telomere length would also be associated with telomere length changes, telomere length at a given timepoint is the outcome of both initial telomere length and subsequent attrition/elongation, which may not be associated. Indeed, in our supplementary analysis (Supplementary Analysis 2) the initial length of telomeres (at day 3) did not affect the rate of telomere length change across early life after controlling for effects of regression to the mean (Verhulst et al., 2013) (however, other studies have detected this trend (Salomons et al., 2009; Aviv et al., 2009)). Hence, variation in initial telomere length, which may be more influenced by heritable genetic factors (Broer et al., 2013, but see Voillemot et al., 2012), may confound the relationship between environmental factors associated with telomere length versus telomere length change, and indeed, may confound the accuracy of telomere length as a biomarker of individual condition later in life (Boonekampp et al., 2014; Wood \& Young 2019). In line with this, in our study we found that genetic and/or familial effects (represented by nest ID) explained the greatest amount of variance in our cross-sectional telomere length measures at both day 3 and day 11, while telomere length change was better described by DNAm changes and growth rate, factors that are extremely sensitive to the environment. 
Contrary to other studies (Stier et al., 2020; Fitzpatrick et al., 2019), we did not find an effect of ambient temperature on telomere length or telomere length changes across development. Temperature has been shown to effect within individual changes in DNAm (Sheldon et al., 2020) and telomere length (Stier et al 2020; Fitzpatrick et al 2019) however, it is unclear if temperature effects these DNA modifications comparably. The temperatures (average daily maximum of $27.6^{\circ} \mathrm{C}$ ) in our study may not have been 'stressful' enough to impact telomere dynamics, and indeed temperature has not affected telomere length in studies on other taxa (McLennan et al., 2018; Boonekamp et al, 2020).

In our longitudinal analyses, we also detected a significant, negative relationship between tarsus growth and change in telomere length, such that individuals that grew faster lost more telomere length across the measured period of their early life. This relationship has been detected in previous studies, and could be due to increases in cell division, energy expenditure and oxidative stress associated with increased growth rate (Monaghan \& Ozanne, 2018). The relationship between telomere length and tarsus growth occurred independently of temperature in our study. However, previous studies have shown that embryonic growth rate, manipulated by small variations $\left(+-1^{\circ} \mathrm{C}\right)$ in incubation temperature, negatively affect telomere length in the laboratory (Stier et al., 2020). We did not detect a relationship between cross-sectional measures of telomere length and tarsus length in our attempt to explore proximate effects within individuals. However, in a study examining the same relationship (between telomere length and tarsus length) at an ultimate level, across generations, in a selection experiment on body size in the house sparrow, a relationship was detected (Ringsby et al., 2015).

In our cross-sectional analysis we detected a positive correlation between telomere length at day 3 and clutch size. We have previously shown that DNAm was affected by brood size during early life of wild zebra finches (Sheldon et al., 2018), and experimental work has also shown that telomeres are generally shorter when brood size is increased during early life (Voillemot et al., 2012; Reichert et al., 2014), potentially as a response to the stress of lower per capita food delivery rates to offspring (Costanzo et al., 2016). The positive relationship detected in our analysis is thus interesting and may reflect higher quality parental care (Bichet et al., 2020), lower rates of nestling/egg mortality, or optimal environmental factors among chicks developing in larger (unmanipulated) clutches (Van Noordwijk \& de Jong, 1986).

In our study, telomere length was found to increase as well as decrease from day 3 to day 11 of post-hatch development. Increasing evidence from a range of avian taxa suggests this could be a somewhat common occurrence (Wood \& Young, 2019; Kotrschal et al., 2007; Young et al., 2013; Uivari \& Madsen, 2009; Haussmann \& Mauck, 2008) possibly due to the actions of telomerase (Haussmann, Winkler, Huntington, Nisbet, \& Vleck, 2004; 2007; Jaskelioff et al., 2011; Bateson \& Nettle, 2017; Hoelzl et al., 2016). The extent to which mechanisms underlying telomere length elongation may weaken the utility of within-individual telomere length variation and, indeed, the utility of telomere length as a biomarker of biological ageing, is unclear, as telomere repair by telomerase may itself be inhibited by the same factors effecting telomere length (e.g. oxidative damage; Ahmed et al. 2008).

Our measurements of absolute telomere length in wild zebra finches gave telomere lengths similar to those in captive birds measured by the same in-gel TRF method; measurements in the same lab and using the same equipment and protocol for laboratory birds (varying in age from 7 to 120 days) ranged from 10.1 to $16.35 \mathrm{~kb}$ (Millet \& Salmon, pers comm). These data suggest that, in contrast to the laboratory mouse which typically has telomeres several times longer than its wild counterparts possibly due to inbreeding (Manning et al 2002), but this is not the case for the zebra finch.

In conclusion, our study of wild zebra finches has confirmed previously documented effects in captive zebra finches of both growth rate and clutch size on telomere length dynamics during early life. This consistency is important given the differences in resources and variability in the environment of wild compared to captive animals. Most significantly, our study detected a negative relationship between early life telomere and DNAm dynamics, such that individuals with accelerated rates of telomere shortening have associated increases in DNAm levels. The nature of this relationship warrants further investigation since it may shed light on potentially clinically/ecologically distinct aspects of biological age reflected by DNAm and telomere 
length. It would be valuable for future work to focus on next-generation sequencing techniques (e.g., reduced representative bisulfite sequencing (RRBS)) to compare DNAm and telomere length measures of biological age across different environments and/or to compare potentially functional relevant or regulatory associations between DNAm and telomere length across different environments. Our results provide a base for further investigations to link and/or disentangle the relationship between the early life environment and DNA biomarkers of biological age.

\section{ACKNOWLEDGMENTS}

We would like to acknowledge funding from an Australian Research Council grant (DP170103619) to SCG and PM. Thanks also to Caroline Millet and Pablo Salmon for the TRF measurements.

\section{REFERENCES}

Ahmed, S., Passos, J. F., Birket, M. J., Beckmann, T., Brings, S., Peters, H., .. \& Saretzki, G. (2008). Telomerase does not counteract telomere shortening but protects mitochondrial function under oxidative stress. Journal of Cell Science, 121, 1046-1053.

Angers, B., Castonguay, E., \& Massicotte, R. (2010) Environmentally induced phenotypes and DNA methylation: how to deal with unpredictable conditions until the next generation and after. Molecular Ecology , 19, 1283-1295.

Aviv A., Chen, W., Gardner, J.P., Kimura, M., Brimacombe, M., Cao, X., . . Berenson, G.S. (2009). Leukocyte telomere dynamics: longitudinal findings among young adults in the Bogalusa Heart Study. American Journal of Epidemiology. 169, 323-329

Bates, D., Machler, M., Bolker, M., \& Walker, S.C. (2015). Fitting linear mixed-effects models using lme4. Journal of Statistical Software, 67, 1-48

Bateson, M., \& Nettle, D. (2016). The telomere lengthening conundrum - it could be biology. Aging Cell , 16(2), 312-319.

Bichet, C., Bouwhuis, S., Bauch, C., Verhulst, S., Becker, P.H., \& Vedder, O. (2020) Telomere length is repeatable, shortens with age and reproductive success, and predicts remaining lifespan in a long-lived seabird. Molecular Ecology, 29, 429-441.

Blackburn, E.H. (1991) Structure and function of telomeres.Nature, 350, 569-573.

Blackburn, E.H., \& Epel, E.S. (2012) Telomeres and adversity: Too toxic to ignore. Nature, 490, 169-171.

Blasco, M.A. (2007) The epigenetic regulation of mammalian telomeres.Nature Reviews Genetics , 8, 299309.

Boonekamp, J.J., Mulder, G., Salomons, H.M., Dijkstra, C., \& Verhulst, S. (2014) Nestling telomere shortening, but not telomere length, reflects developmental stress and predicts survival in wild birds.Proceedings of the Royal Society B: Biological Sciences, 281 (1785), 20133287.

Boonekamp, J., Rodriguez-Munoz, R., Hopwood, P, Zuidersma, E., Mulder, E., Wilson, A., .. \& \& Tregenza, T. (2020) Telomere length is highly heritable and independent of growth rate manipulated by temperature in field crickets. bioRxiv,doi/10.1101/2020.05.29.123216.

Broer, L., Codd, V., Nyholt, D.R., Deelen, J., Mangino, M., Willemsen, G., .. \& Boomsma DI. (2013). Meta-analysis of telomere length in 19,713 subjects reveals high heritability, stronger maternal inheritance, and a paternal age effect. European Journal of Human Genetics . 21(10),1163-1168.

Buxton, J., Suderman, M., Pappas, J., Borghol, N., McArdle, W., Blakemore., .. \& Pembrey, M. (2014). Human leukocyte telomere length is associated with DNA methylation levels in multiple subtelomeric and imprinted loci. Scientific Reports, 4, 4954, doi.org/10.1038/srep04954 
Costanzo, A., Parolini, M., Bazzi, G., Khoriauli, L., Santagostino, M., Possenti, C.D., . . \& Saino, N. (2016) Brood size, telomere length, and parent-offspring color signaling in barn swallows.Behavioral Ecology, 28, 204-211.

de Lange, T. (2005) Shelterin: the protein complex that shapes and safeguards human telomeres. Genes and Development ,19, 2100-2110

Dong, Y., Huang, Y., Gutin, B., Raed, A., Dong, Y., \& Zhu, H. (2017) Associations between Global DNA Methylation and Telomere Length in Healthy Adolescents. Scientific Reports , 7, 4210, doi.org/10.1038/s41598-017-04493-z

Dugdale, H.L., \& Richardson, D.S. (2018) Heritability of telomere variation: it is all about the environment! Philosophical Transactions of the Royal Society B , 373 (1741), doi: 10.1098/rstb.2016.0450

Eastwood, J.R., Hall, M.L., Teunissen, N., Kingma, S.A., Hidalgo Aranzamendi, N., Fan M., .. \& Peters, A. (2019). Early-life telomere length predicts lifespan and lifetime reproductive success in a wild bird. Molecular Ecology : 28(5):1127-1137.

Feil, R., \& Fraga, M. E. (2012). Epigenetics and the environment: emerging patterns and implications. Nature Review Genetics .13: 97-109.

Fitzpatrick, L.J., Olsson, M., Parsley, L.M. et al. (2017). Temperature and telomeres: thermal treatment influences telomere dynamics through a complex interplay of cellular processes in a cold-climate skink. Oecologia 191, 767-776. https://doi.org/10.1007/s00442-019-04530-w

Gilby, A.J., Mainwaring, M.C., \& Griffith, S.C. (2013) Incubation behaviour and hatching synchrony differ in wild and captive populations of the zebra finch. Animal Behaviour, 85, 1329-1334.

Gonzalo, S., Jaco, I., Fraga, M.F., Chen, T., Li, E., Esteller, M., \& Blasco, M.A. (2006) DNA methyltransferases control telomere length and telomere recombination in mammalian cells. Nature Cell Biology,8, 416-424.

Griffith, S. C., Pryke, S. R. \& Mariette, M. (2008). Nest box use by the zebra finch Taeniopygia guttata: implications for reproductive success and research. Emu, 108: 311-319.

Harley, C.B., Futcher, A.B., Greider, C.W. (1990). Telomeres shorten during ageing of human fibroblasts. Nature , 345(6274):458-460

Haussmann, M. F., Winkler, D. W., Huntington, C. E., Nisbet, I. C. T., \& Vleck, C. M. (2004). Telomerase expression is differentially regulated in birds of differing life span. Annals of the New York Academy of Sciences , 1019, 186-190.

Haussmann, M. F., Winkler, D. W., Huntington, C. E., Nisbet, I. C. T., \& Vleck, C. M. (2007). Telomerase activity is maintained throughout the lifespan of long-lived birds. Experimental Gerontology , 42, 610-618

Haussmann, M.F., Mauck, R.A. (2008). Telomeres and longevity: testing an evolutionary hypothesis. Molecular Biology and Evolution, 25: 220-228.

Hemann, M.T., Strong, M.A., Hao, L.Y., Greider, C.W. (2001). The shortest telomere, not average telomere length, is critical for cell viability and chromosome stability. Cell . 107(1), 67-77.

Hoelzl, F., Smith, S., Cornils, J. S., Aydinonat, D., Bieber, C., \& Ruf, T. (2016). Telomeres are elongated in older individuals in a hibernating rodent, the edible dormouse (Glis glis ). Scientific Reports , 6 , 36856. doi.org/10.1038/srep36856

Hornsby, P.J. (2003) Replicative senescence of human and mouse cells in culture: significance for aging research. Mechanisms of Ageing and Development, 124, 853-855.

Horvath, S. (2013). DNA methylation age of human tissues and cell types.Genome Biology . 14(10), doi: 10.1186/gb-2013-14-10-r115. 
Horvath, S., Raj, K. (2018). DNA methylation-based biomarkers and the epigenetic clock theory of ageing. Nature Reviews Genetics . 19 (6), 371-384.

Hoelzl, F., Smith, S., Cornils, J. S., Aydinonat, D., Bieber, C., \& Ruf, T. (2016). Telomeres are elongated in older individuals in a hibernating rodent, the edible dormouse (Glis glis ). Scientific Reports , 6 , 36856, doi.org/10.1038/srep36856

Jaskelioff, M., Muller, F. L., Paik, J. H., Thomas, E., Jiang, S., Adams, A. C., .. \& Depinho, R.A. (2011). Telomerase reactivation reverses tissue degeneration in aged telomerase-deficient mice. Nature , 469 , $102-106$.

Jimeno, B., Hau, M., Gómez-Díaz, E., Verhulst, S. (2019). Developmental conditions modulate DNA methylation at the glucocorticoid receptor gene with cascading effects on expression and corticosterone levels in zebra finches. Scientific Reports, 9, doi.org/10.1038/s41598-019-52203-8

Jylhava, J., Pedersen, N. L., \& Hagg, S. (2017). Biological age predictors. EBioMedicine , 21, 29-36.

Kotrschal, A., Ilmonen, P., Penn, D.J. (2007). Stress impacts telomere dynamics. Biology Letters, 3, 128-130.

Kuznetsova, A., Brockhoff, P. B., \& Christensen, R. H. B. (2016). lmerTest package: tests in linear mixed effects models. Journal of Statistical Software, 82 (13), 1 - 26.

Banszerus, V.L., Vetter, V.M., Salewsky, B., König, M., Demuth, I. 2019. Exploring the Relationship of Relative Telomere Length and the Epigenetic Clock in the LipidCardio Cohort. International Journal of Molecular Sciences , 20(12):3032. doi.org/10.3390/ijms20123032

Lee, Y., Sun, D., Ori, A., Lu, A. T., Seeboth, A., Harris. J.R., .. \& Horvath, S. (2019). Epigenome-wide association study of leukocyte telomere length. Aging , 11(16), 5876-5894.

Lu, A. T., Seeboth, A., Tsai, P. C., Sun, D., Quach, A., Reiner, A. P., .. \& Horvath, S. (2019). DNA methylation-based estimator of telomere length. Aging , 11(16), 5895-5923.

Mariette, M. M., \& Griffith, S. C. (2015). The adaptive significance of provisioning and foraging coordination between breeding partners. The American Naturalist , 185, 270-280.

Makinen, H., van Oers., Eeva, T., Laine, V.N., Ruuskanen, S. 2020. The effect of experimental lead pollution on DNA methylation in a wild bird population. bioRxiv 851998; doi: https://doi.org/10.1101/851998

McLennan, D., Armstrong, J.D., Stewart, D.C., Mckelvey, S., Boner,W., Monaghan,P., \& Metcalfe, N.B. (2018) Telomere elongation during early development is independent of environmental temperatures in Atlantic salmon. The Journal of Experimental Biology, 221, doi,10.1242/jeb.178616.

Monaghan, P. (2010) Telomeres and life histories: the long and the short of it. Annals of the New York Academy of Sciences, 1206, 130-142.

Monaghan, P. (2014). Organismal stress, telomeres and life histories. Journal of Experimental Biology, 217, $57-66$.

Monaghan, P., \& Ozanne, S.E. (2018). Somatic growth and telomere dynamics in vertebrates: relationships, mechanisms, and consequences.Philosophical Transactions of the Royal Society B: Biological Sciences, 373, 20160446, doi.org/10.1098/rstb.2016.0446.

Nakagawa, S., \& Schielzeth, H. (2013). A general and simple method for obtaining $\mathrm{R}^{2}$ from generalized linear mixed effects models. Methods in Ecology and Evolution , 4, 133-142

Nettle, D., Andrews, C., Reichert, S., Bedford, T., Gott, A., Parker, C., Kolenda, C., Martin-Ruiz, C., Monaghan, P. and Bateson, M. (2016). Brood size moderates associations between relative size, telomere length, and immune development in European starling nestlings.Ecology and Evolution , 6: 8138- 8148. doi: $10.1002 /$ ece3.2551 
Noguera, J., Metcalfe, N., Reichert, S., \& Monaghan, P (2016). Embryonic and postnatal telomere length decrease with ovulation order within clutches. Scientific Reports,6, 25915, doi.org/10.1038/srep25915.

Noguera, J. C., \& Velando, A. (2020). Gull chicks grow faster but lose telomeres when prenatal cues mismatch the real presence of sibling competitors. Proceedings of the Royal Society B , Biological Sciences , 287(1927), doi.org/10.6084/m9.figshare.c.4971167

Nussey, D.H., Baird, D., Barrett, E., Boner, W., Fairlie, J., Gemmell, N., Hartmann, N., Horn, T., Haussmann, M., Olsson, M., Turbill, C., Verhulst, S., Zahn, S. and Monaghan, P. (2014). Measuring telomere length and telomere dynamics in evolutionary biology and ecology.Methods in Ecology and Evolution , 5: 299-310. doi.org/10.1111/2041-210X.12161

Olsson, M., Pauliny, A., Wapstra, E., Uller, T., Schwartz, T., \& Blomqvist, D. (2011). Sex differences in sand lizard telomere inheritance: paternal epigenetic effects increase telomere heritability and offspring survival. PLoS ONE, 6, e17473, doi.org/10.1371/journal.pone.0017473

R Core Team (2017). R: A language and environment for statistical computing. R Foundation for Statistical Computing, Vienna, Austria. URL https://www.R-project.org/.

Reichert, S., Stier, A., Zahn, S., Arrive, M., Bize, P., Massemin, S., \& Criscuolo, F. (2014) Increased brood size leads to persistent eroded telomeres. Frontiers in Ecology and Evolution, 2 (9), doi.org/10.3389/fevo.2014.00009

Richards, C. L., Schrey, A. W., \& Pigliucci, M. (2012). Invasion of diverse habitats by few Japanese knotweed genotypes is correlated with epigenetic differentiation. Ecology Letters , 15: 1016-1025.

Ringsby, T.H., Jensen, H., Parn, H., Kvalnes, T., Boner, W., Gillespie, R., .. \& Monaghan, P. (2015) On being the right size: increased body size is associated with reduced telomere length under natural conditions. Proceedings of the Royal Society B: Biological Sciences, 282 (1820), 20152331, doi.org/10.1098/rspb.2015.2331

Salmon, A., Clotault, J., Jenczewski, E., Chable, V., Manzanares-Dauleux, M. 2008. Brassica oleracea displays a high level of DNA methylation polymorphism. Plant Science, 170 (61)

Salomons, H.M., Mulder, E., van de Zande, L., Haussmann, M.F., Linskens, M., \& Verhulst, S. (2009). Telomere shortening and survival in free-living corvids. Proceedings of the Royal Society B, Biological Sciences $276,3157-3165$.

Schrey, A. W., Alvarez, M., Foust, C. M., Kilvitis, H. J., Lee, J. D., Liebl, A. L., Martin, L. B., Richards, C. L., \& Robertson, M. (2013). Ecological epigenetics: beyond MS-AFLP. Integrative and Comparative Biology , 53(2), 340-50.

Sheldon, E.L., Schrey, A.W., Ragsdale, A., \& Griffith, S.C. (2018). Brood size influences patterns of DNA methylation in wild Zebra Finches (Taeniopygia guttata). The Auk, 135 (4), 1113-1122.

Sheldon, E.L., Schrey, A.W., Hurley, L.L., \& Griffith, S.C. (2020) Dynamic changes in DNA methylation during postnatal development in zebra finches (Taeniopygia guttata) exposed to different temperatures. Journal of Avian Biology, 51 (5), doi.org/10.1111/jav.02294.

Stier, A., Metcalfe, N.B., Monaghan, P. (2020). Pace and stability of embryonic development affect telomere dynamics: an experimental study in a precocial bird model. Proceedings of the Royal Society B , 287 (1933), doi.org/10.1098/rspb.2020.1378.

Tricola, G.M., Simons, M.J., Atema, E., Boughton, R.K., Brown, J.L., Dearborn, D., .. \& \& Haussmann, M. (2018) The rate of telomere loss is related to maximum lifespan in birds. Philosophical Transactions of the Royal Society B: Biological Sciences, 373 (1741), 20160445, doi,10.1098/rstb.2016.0445

Van Noordwijk, A.J., \& de Jong, G. (1986) Acquisition and allocation of resources: their influence on variation in life history tactics. The American Naturalist , 128, 137-142. 
Vedder, O., Verhulst, S., Zuidersma, E., \& Bouwhuis, S. (2018). Embryonic growth rate affects telomere attrition: an experiment in a wild bird. The Journal of Experimental Biology, 221, doi,10.1242/jeb.181586.

Verdun, R.E., \& Karlseder, J. (2007). Replication and protection of telomeres. Nature, 447(7147), 924-31.

Verhulst, S., Aviv, A., Benetos, A., Berenson, G.S., \& Kark, J.D. (2013). Do leukocyte telomere length dynamics depend on baseline telomere length? An analysis that corrects for 'regression to the mean'. European Journal of Epidemiology, 28, 859-866.

Victorelli, S., \& Passos, J. F. (2017). Telomeres and Cell Senescence - Size Matters Not. EBioMedicine, 21 , $14-20$.

Voillemot, M., Hine, K., Zahn, S., Criscuolo, F., Gustafsson, L., Doligez, B., \& Bize, P. (2012). Effects of brood size manipulation and common origin on phenotype and telomere length in nestling collared flycatchers. BMC Ecology, 12, 1-8.

Watson, H., Bolton, M., \& Monaghan, P. (2015). Variation in early-life telomere dynamics in a long-lived bird: links to environmental conditions and survival. The Journal of Experimental Biology , 218 (5), 668-674.

Wilbourn, R.V., Moatt, J.P., Froy, H., Walling, C.A., Nussey, D.H., Boonekamp, J.J. 2018 The relationship between telomere length and mortality risk in non-model vertebrate systems: a meta-analysis. Phiolosophical Transactions Royal Society B, 373: 20160447. doi.org/10.1098/rstb.2016.0447

Wood, E.M., \& Young, A.J. 2019. Telomere attrition predicts reduced survival in a wild social bird, but short telomeres do not.Molecular Ecology . 28 (16), 3669-3680.

Wong, K.K., Maser, R.S., Bachoo, R.M., Menon, J., Carrasco, D.R., Gu, Y., .. \& DePinho, R.A. (2003). Telomere dysfunction and Atm deficiency compromises organ homeostasis and accelerates ageing. Nature . 421(6923), 643-648.

Yan, X., Liu, H., Liu, J., Zhang, R., Wang, G., ...Wang, J. (2015). Evidence in duck for supporting alteration of incubation temperature may have influence on methylation of genomic DNA, Poultry Science , 94 (10), 2537-2545, doi.org/10.3382/ps/pev201.

Young, R.C., Kitaysky, A.S., Haussmann M.F., Descamps, S., Orben, R.A., Elliott, K.H., \& Gaston, A.J. (2013). Age, Sex, and telomere dynamics in a long-lived seabird with male biased parental care. PLoS One , 8(9), 24023967, doi.org/10.1371/journal.pone.0074931.

Young, R.C., Welcker, J., Barger, C.P., Hatch, S.A., Merkling, T., Kitaiskaia, E.V., .. Kitaysky, A,S. (2017). Effects of developmental conditions on growth, stress and telomeres in black-legged kittiwake chicks. Molecular Ecology . 26(13), 3572-3584. doi: 10.1111/mec.14121.

\section{DATA ACCESSIBILITY}

Authors have archived their data in the publicly accessible repository FigShare. DOI: 10.6084/m9.figshare.13670044

\section{STATEMENT OF CONFLICTING INTEREST}

The authors declare no conflict of interest.

\section{AUTHOR CONTRIBUTIONS}

E.L.S., P.M. and S.C.G. conceived the study. E.L.S. collected the field data. E.L.S. and A.W.S. performed the MS-AFLP assay. W.B., R.T., S.R., and P.M. performed the qPCR assay. E.L.S. and R.T. conducted the statistical analyses. The manuscript was written by E.L.S. and commented on and edited by all authors. S.C.G provided funding.

\section{AUTHOR ORCID I.D.}

Elizabeth L. Sheldon https://orcid.org/0000-0001-5002-3006 
Riccardo Ton https://orcid.org/0000-0003-3897-9720

Simon C. Griffith https://orcid.org/0000-0001-7612-4999

Aaron. W. Schrey https://orcid.org/0000-0002-2165-7349

Pat Monaghan https://orcid.org/0000-0003-2430-0326;

Winnie Boner https://orcid.org/0000-0003-1702-4298

Shirley Raveh https://orcid.org/0000-0003-0406-4928

\section{TABLES AND FIGURES}

Figure 1 . Conceptual description of the associations between an environmental variable (ambient temperature), brood size, growth, DNAm and telomere length. An exemplar citation is provided as an example of a study exploring each association.

\section{Hosted file}

image1.emf available at https://authorea.com/users/404965/articles/516046-associationsbetween-dna-methylation-and-telomere-length-during-early-life-insight-from-wild-zebrafinches-taeniopygia-guttata

Figure 2 . Paired box plot illustrating individual changes in telomere length across early life (from day 3 to day 11 post-hatch) for 50 individuals from 38 broods. Grey lines connect individuals across development. The box plots show the median, lower, and upper quartile of telomere length data. 


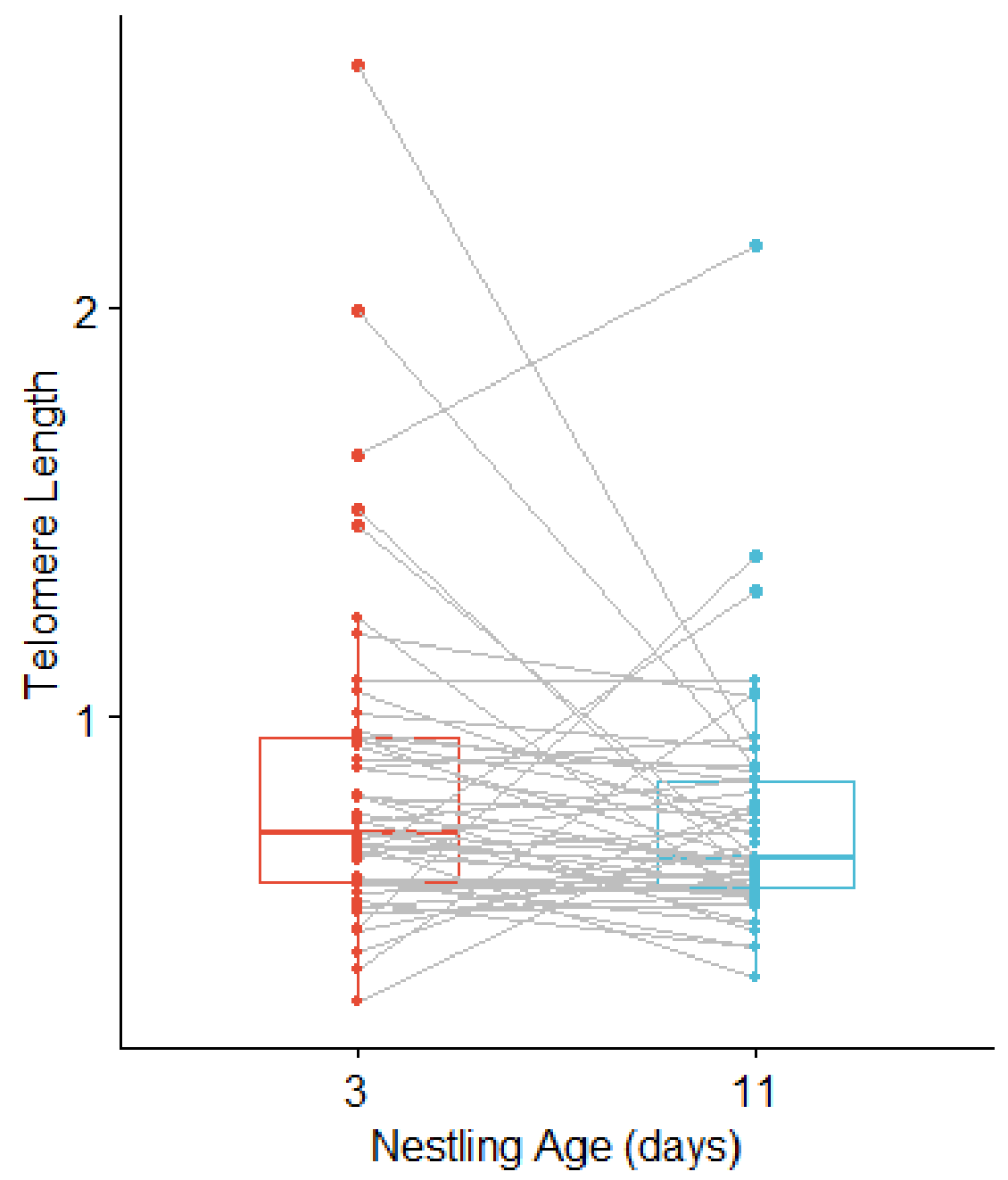

Table 1 : Random and fixed effect estimates from a linear mixed model for change in telomere length $(n$ $=24$ individuals from 20 nests) from day 3 to day 11 post-hatch. Significant values are indicated by $*$ at the $95 \%$ confidence interval. $\mathrm{SE}=$ standard error.

Model 1. Dependent Variable: Early life change in telomere length

\begin{tabular}{lllll}
\hline Fixed effect & Estimate & SE & t-value & $p$-value \\
Change in Tarsus Length & -1.499 & 0.580 & -2.397 & $0.021^{*}$ \\
Change in DNA methylation (\%) & -0.875 & 0.397 & -2.126 & $0.044^{*}$ \\
Post-hatch Temperature & 0.037 & 0.026 & 0.732 & 0.255 \\
Tarsus Length (day 3) & 0.388 & 0.264 & 1.469 & 0.164 \\
Brood Size & $>0.001$ & 0.083 & -0.566 & 0.999 \\
Random effect & Variance & St Dev & & \\
Nest ID & 0.002 & 0.042 & & \\
Residual & 0.263 & 0.513 & & \\
\hline
\end{tabular}

Figure 3a . Partial residual plot illustrating the relationship between changes in DNA methylation levels 
(\%) (x-axis) and telomere length (y-axis) from day 3-11 post-hatch while controlling for differences in tarsus growth, post-hatch temperature, and brood size.

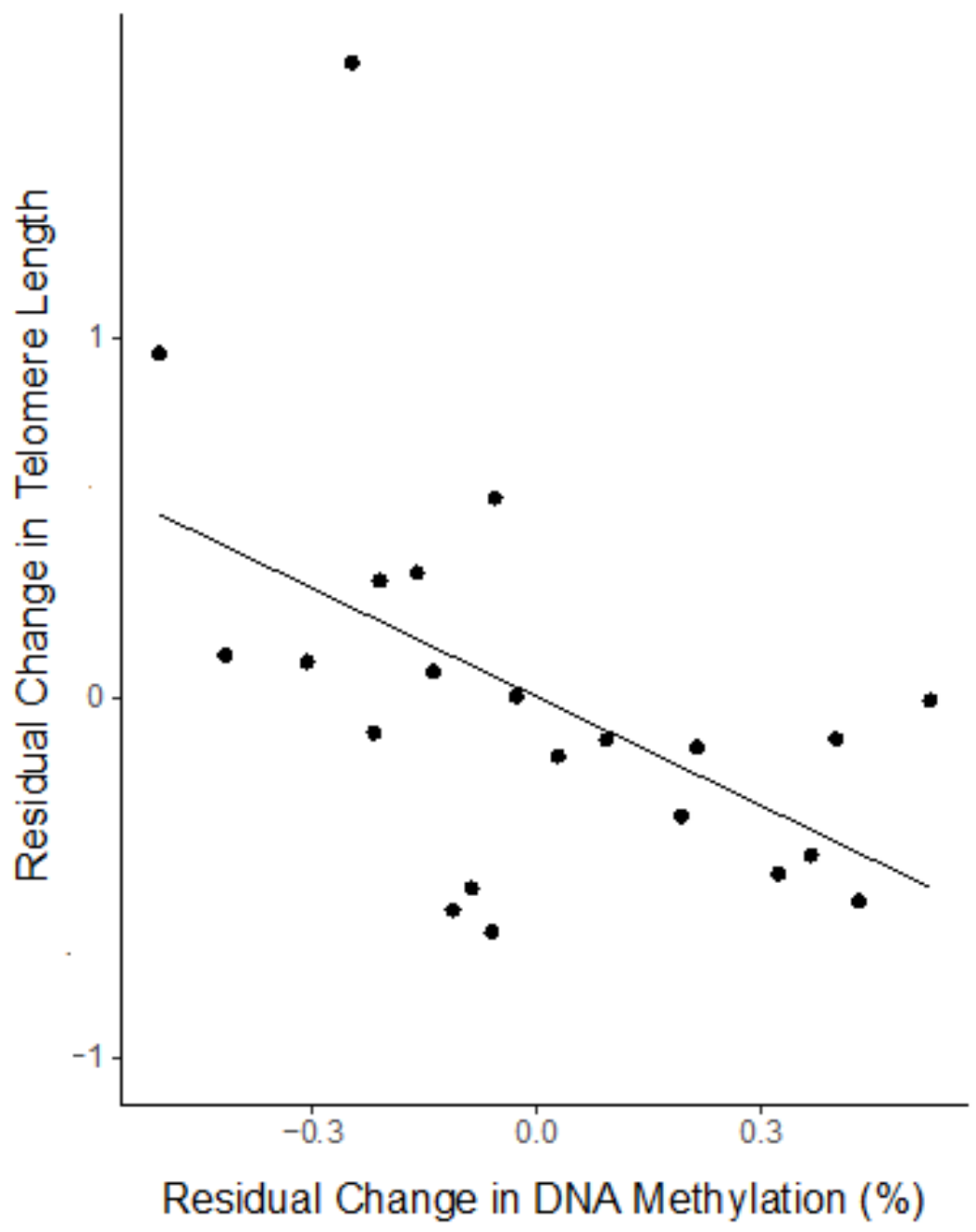

Figure $3 \mathrm{~b}$. Partial residual plot illustrating the relationship between tarsus growth (x-axis) and telomere length change (y-axis) from day 3-11 post-hatch while controlling for changes in DNA methylation levels (\%), post-hatch temperature, and brood size. 


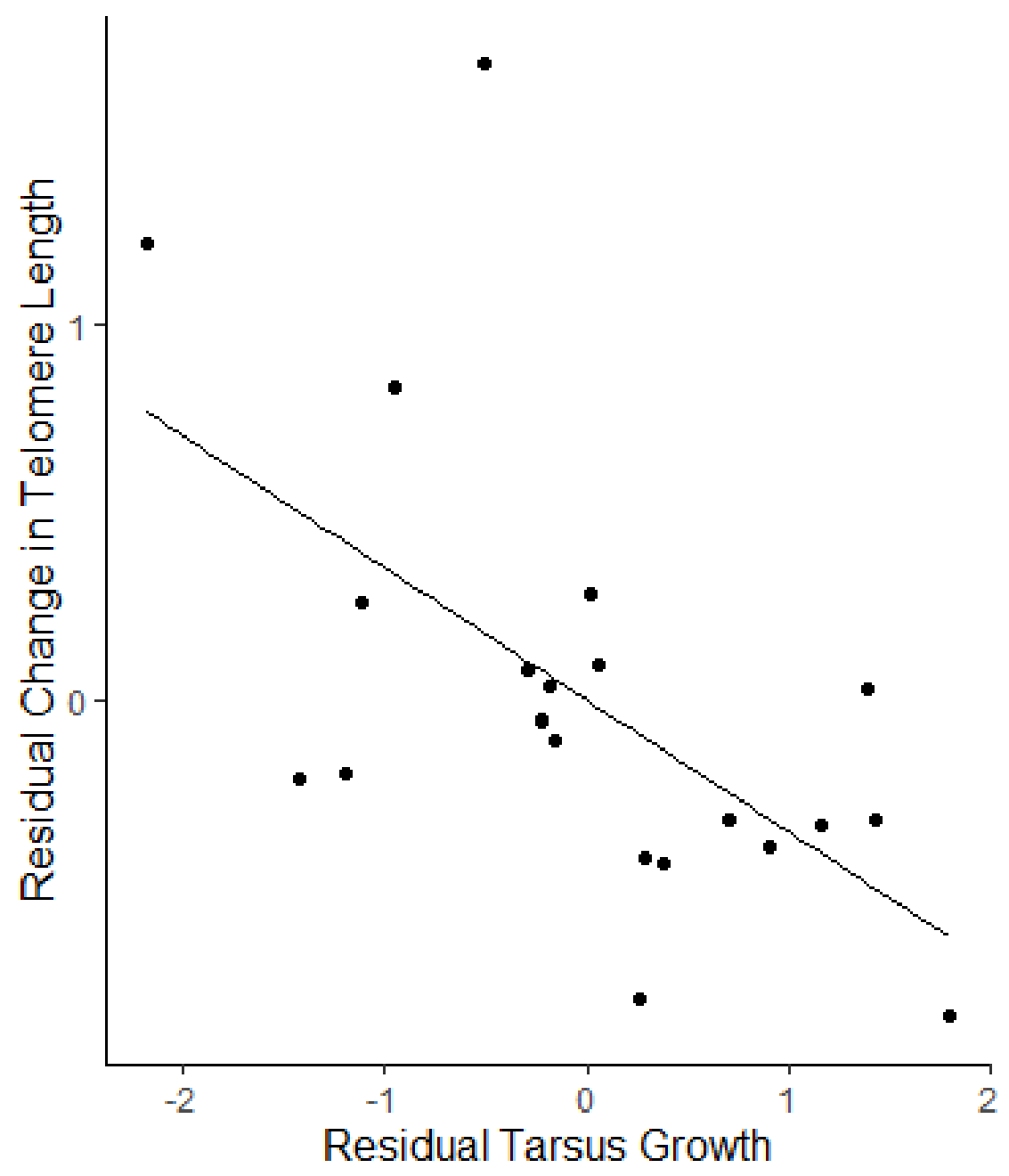

Table 2. Random and fixed effect estimates from a linear mixed model for telomere length at day $3(\mathrm{n}=$ 33 individuals from 26 nests). Significant values are indicated by $*$ at the $95 \%$ confidence interval. SE $=$ Standard error.

Model 3. Dependent Variable: Telomere length at day 3

\begin{tabular}{lllll}
\hline Fixed effect & Estimate & SE & t-value & $p$-value \\
Tarsus Length (day 3) & -0.132 & 0.113 & -1.168 & 0.253 \\
DNA methylation (\%) (day 3) & -1.787 & 1.105 & -1.617 & 0.117 \\
Incubation Temperature & 0.014 & 0.020 & 0.678 & 0.503 \\
Clutch Size & 0.104 & 0.114 & 2.275 & $0.032^{*}$ \\
Random effect & Variance & St Dev & & \\
Nest ID & 0.093 & 0.301 & & \\
Residual & 0.048 & 0.221 & & \\
\hline
\end{tabular}

Table 3 . Random and fixed effect estimates from a linear mixed model for telomere length at day $11(n$ $=28$ individuals from 23 nests). Significant values are indicated by $*$ at the $95 \%$ confidence interval. $\mathrm{SE}=$ 
Standard error.

Model 1. Dependent Variable: Telomere length at day 11

\begin{tabular}{lllll}
\hline Fixed effect & Estimate & SE & t-value & $p$-value \\
Tarsus Length (day 11) & 0.001 & 0.077 & 0.017 & 0.987 \\
DNA methylation (\%) (day 11) & 0.080 & 0.786 & 0.102 & 0.920 \\
Post-hatch Temperature & 0.022 & 0.016 & 1.337 & 0.197 \\
Brood Size & -0.031 & 0.053 & -0.589 & 0.564 \\
Random effect & Variance & St Dev & & \\
Nest ID & 0.091 & 0.301 & & \\
Residual & 0.048 & 0.221 & & \\
\hline
\end{tabular}

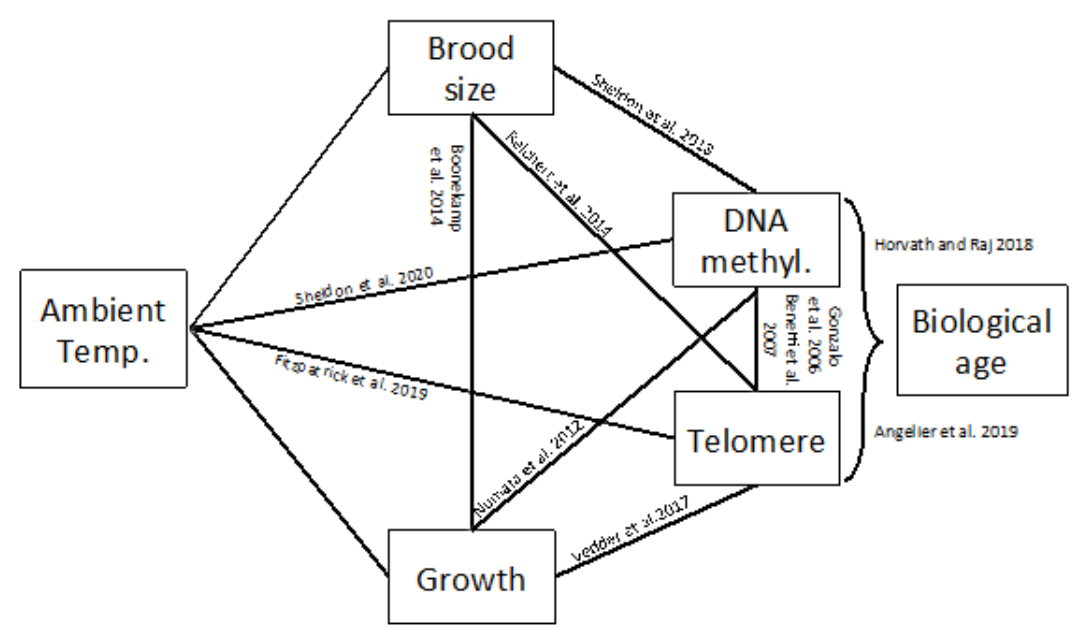




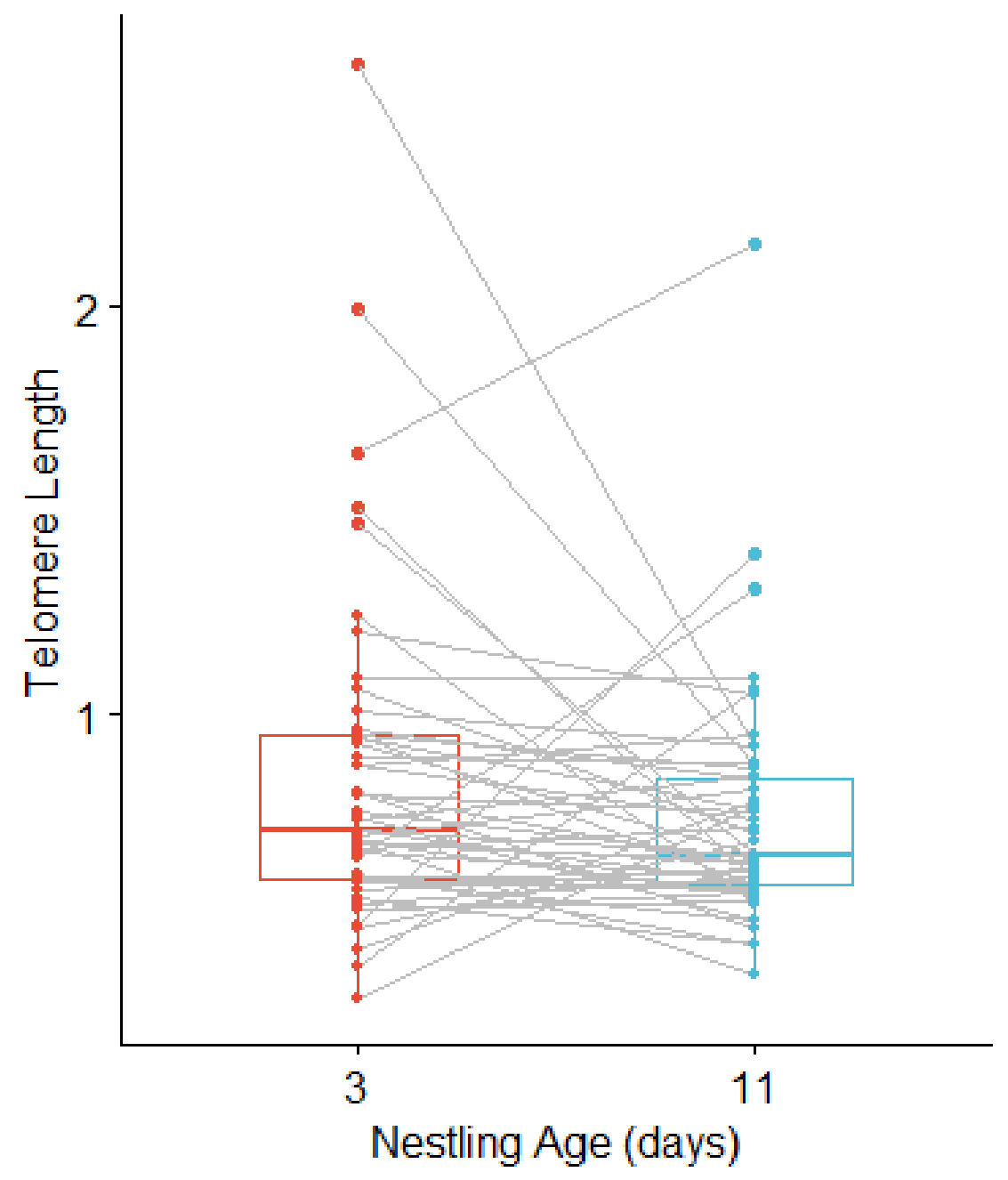




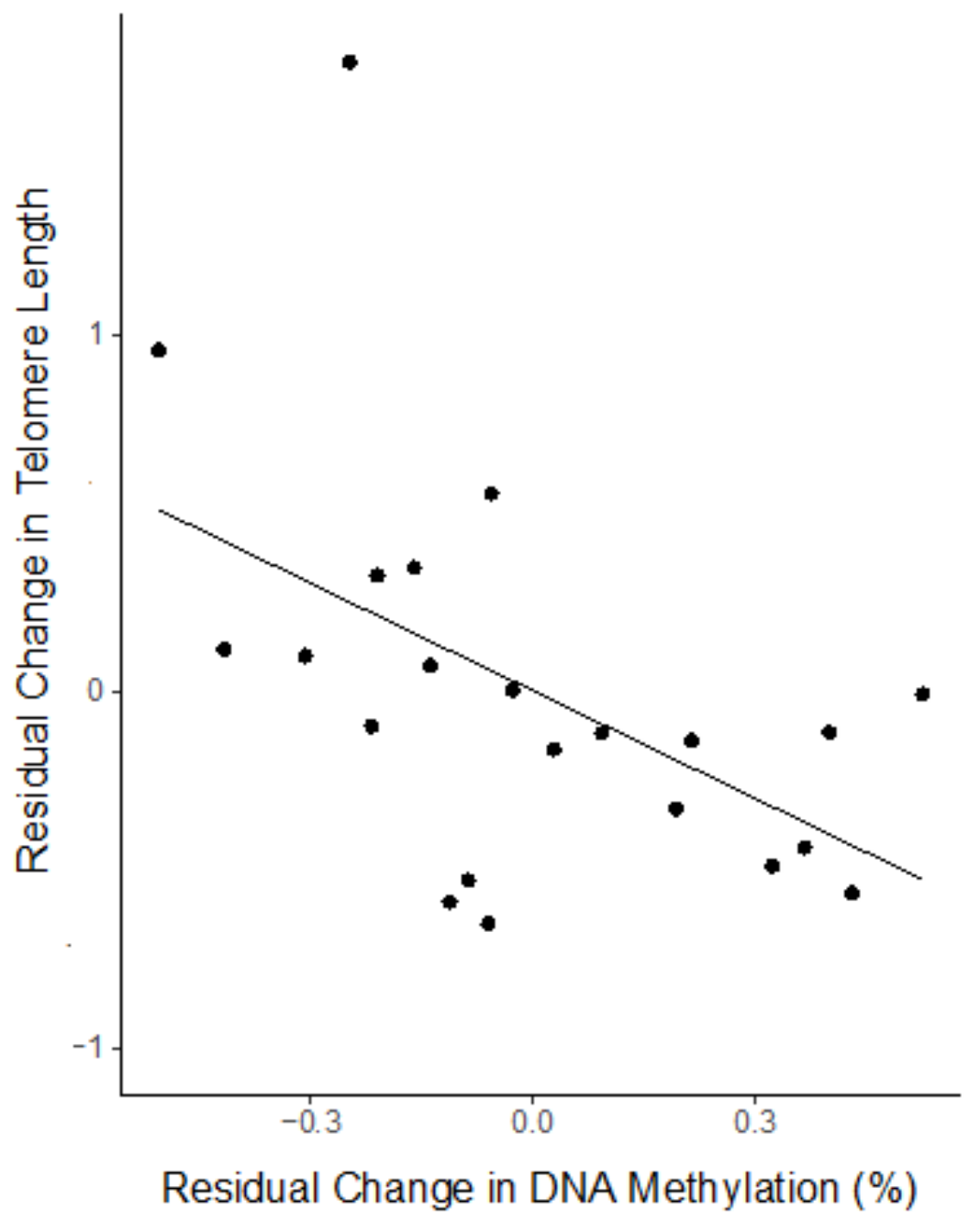




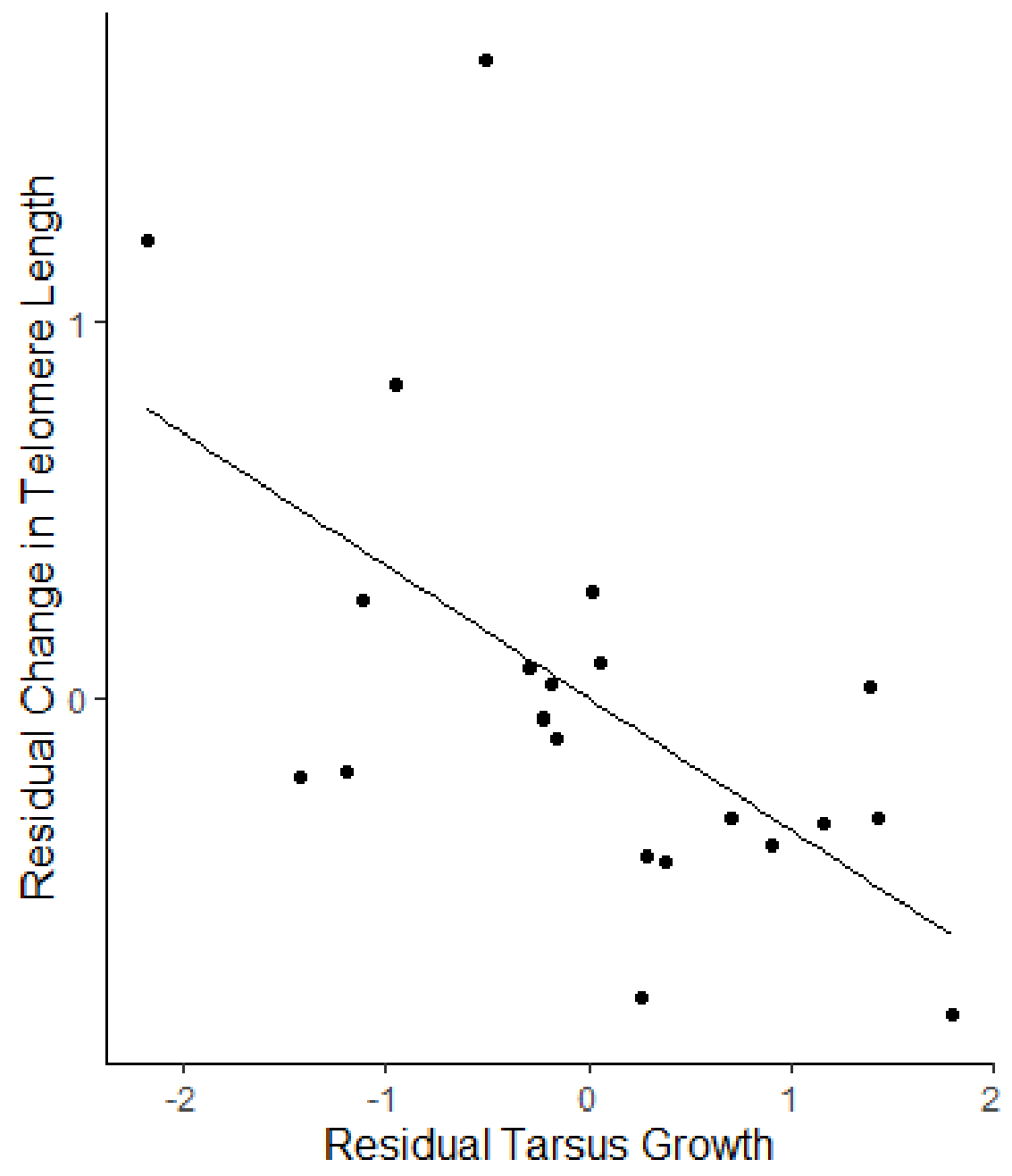

